\title{
Never Miss Sleep Disorder in Children with Drop Attacks
}

\author{
Madhan Kumar $^{1} \cdot$ Sangeetha Yoganathan ${ }^{2} \cdot$ Murugan T. $\mathrm{P}^{1} \cdot$ Maya Mary Thomas ${ }^{2}$
}

Received: 7 May 2021 / Accepted: 10 June 2021 / Published online: 1 September 2021

(c) Dr. K C Chaudhuri Foundation 2021

To the Editor: Drop attacks refer to sudden falls without impairment of awareness or consciousness followed by complete recovery [1]. Causes of drop attacks are myoclonic seizures, atonic seizures, movement disorders, sleep disorders, and psychiatric disorders [1]. An 8-y-old girl presented with excessive daytime sleepiness (EDS), multiple drop attacks precipitated by laughter and scholastic deterioration. She was treated elsewhere with antiepileptic medications without any clinical response. There was no facial dysmorphism, tonsillar enlargement or focal neurological deficit. Possibilities of subacute sclerosing panencephalitis, progressive myoclonic epilepsy, and narcolepsy were considered. Magnetic resonance imaging of brain and electroencephalography were normal. $H L A-D Q B 1$ was identified. Cerebrospinal fluid (CSF) and serum antimeasles antibody titre was not significant. Polysomnography (PSG) revealed a mean sleep latency of $7.7 \mathrm{~min}$ on multiple sleep latency test (MSLT) and two sleep onset rapid eye movement period (SOREMPs) confirming the diagnosis of narcolepsy. Sleep hygiene measures were emphasized and the child was treated with modafinil and venlafaxine following which there was a significant clinical improvement.

Narcolepsy is characterized by EDS and at least one of following features such as cataplexy, CSF hypocretin deficiency, night PSG revealing a rapid eye movement sleep latency $\leq 15 \mathrm{~min}$, or MSLT confirming a mean sleep latency $\leq 8$ min with $\geq 2$ SOREMPs [2]. Sleep disorders in children may be screened using "BEARS $(\mathrm{B}=$ Bedtime Issues, $\mathrm{E}=$ Excessive Daytime Sleepiness, $\mathrm{A}=$ Night Awakenings, $\mathrm{R}=$ Regularity and Duration of Sleep, $\mathrm{S}=$ Snoring)" sleep screening tool [3]. Treatment of narcolepsy includes behavioural modification such as scheduled daytime naps and

Maya Mary Thomas

maya@cmcvellore.ac.in

1 Department of Pediatrics, Christian Medical College, Vellore, Tamil Nadu 632004, India

2 Pediatric Neurology Unit, Department of Neurological Sciences, Christian Medical College, Vellore,

Tamil Nadu 632004, India short naps before activities demanding high attention span and pharmacotherapy. EDS may be managed with sodium oxybate, dextroamphetamine, methylpehnidate, modafinil, armodafinil, and pitolisant [4]. Drugs used in management of cataplexy are sodium oxybate, clomipramine, imipramine, fluoxetine, and zimelidine [4]. Though epilepsies remain the common cause of drop attacks in children, other causes such as cataplexy and movement disorders should not be missed. A high index of clinical suspicion with emphasis on sleep history often helps in early diagnosis and management of narcolepsy.

\section{Declarations}

Conflict of Interest None.

\section{References}

1. Rosati A, Berti B, Melani F, Cellini E, Procopio E, Guerrini R. Recurrent drop attacks in early childhood as presenting symptom of benign hereditary chorea caused by TITF1 gene mutations. Dev Med Child Neurol. 2015;57:777-9.

2. Ruoff C, Rye D. The ICSD-3 and DSM-5 guidelines for diagnosing narcolepsy: clinical relevance and practicality. Curr Med Res Opin. 2016;32:1611-22.

3. Owens JA, Dalzell V. Use of the 'BEARS' sleep screening tool in a pediatric residents' continuity clinic: A pilot study. Sleep Med. 2005;6:63-9.

4. Lammers GJ. Drugs used in narcolepsy and other hypersomnias. Sleep Med Clin. 2018;13:183-9.

Publisher's Note Springer Nature remains neutral with regard to jurisdictional claims in published maps and institutional affiliations. 